Conclusion: The study is ongoing. Preliminary data suggest that a significant proportion of RTA victims do not arrive by EMS. We hope to review the data to determine if EMS transport confers advantage to crash victims and advise changes to improve EMS-based outcomes.

Prehosp Disaster Med 2017;32(Suppl. 1):s164-s165

doi:10.1017/S1049023X17004435

Prehospital Care of Spinal Cord Injuries in India Kasha Rogers-Smith ${ }^{1}$, Chris Turner ${ }^{2}, H$ S. Chbabra ${ }^{3}$

1. Warwick Medical School, Coventry/United Kingdom

2. UHCW, Coventry/United Kingdom

3. Indian Spinal Injuries Centre, New Delhi/India

Study/Objective: Prehospital Care of Spinal Cord Injuries in India.

Background: Injury is an increasing burden in Low and Middle Income Countries (LMICs) including in India. Prehospital care refers to the initial medical care given to a patient following injury, and before they present to a hospital. As in several developing countries, India lacks an established prehospital care system. Appropriate prehospital care is especially pertinent for a patient with a suspected Spinal Cord Injury (SCI), as effective immobilization and transportation is vital to avoid secondary injury.

Methods: Interviews were conducted at the 145-bed tertiary-level hospital, Indian Spinal Injuries Center in New Delhi, between March 10, 2016 and October 10, 2016 using a pre-designed questionnaire. Inclusion criteria was any patient $>16$ years who had suffered a SCI in India. Patients $<16$ years and who had suffered their injury outside of India, were excluded from the study. Interviews were conducted in the Rehabilitation Department with inpatients and outpatients attending for physiotherapy.

Results: Overall, $53.33 \%$ of SCI was caused by road traffic accidents and $26.67 \%$ were due to a fall from height; $50.00 \%$ of patients were transported to hospital by ambulance, with a median transfer time of 2.5 hours. The remaining $50 \%$ of patients were transported by private car $(26.67 \%)$, auto-rickshaw (6.67\%), police car (6.67\%), taxi (3.33\%) or bus (3.33\%). Further, $33.33 \%$ of patients transported by ambulance received pain relief, and $26.67 \%$ were transported with a neck collar or on a back board. Overall $16.67 \%$ of patients received pre-hospital care. Conclusion: As the burden of injuries, in particular those caused by road traffic accidents rises, India is increasingly in need of a country-wide, established prehospital care system. In the last decade, ambulance use has increased, but there are huge inconsistencies in the care ambulance staff provide. In addition, awareness of the identification and management of SCI needs to raised among both health care professionals and lay persons. Prehosp Disaster Med 2017;32(Suppl. 1):s165

doi:10.1017/S1049023X17004447

\section{Cardiac Arrest, Are Two ALS Providers Better than One?} Kelly R. Klein ${ }^{1}$, Carla Cash ${ }^{2}$

1. Emergency Medicine, UT Southwestern Medical Center at Dallas, Dallas/United States of America

2. Emergency Medicine, UT Southwestern Medical Center at Dallas, Dallas/TX/United States of America
Study/Objective: To determine if, in our geographic area of the US, the use of a duel paramedic Emergency Medical Service (EMS) system is a factor in improving cardiac arrest prehospital care, or should a single paramedic system be adapted.

Background: Among many urban EMS systems, there exists a paradigm of belief regarding dual ALS provider ambulances, that more advanced training must equal better care. Though much research has focused on the benefits of Advanced Life Support (ALS) versus Basic Life Support (BLS), far fewer studies have been devoted to whether there is any true benefit of dual ALS ambulances. Since 1966 and the publishing of Accidental Death and Disability: The Neglected Disease of Modern Society by the National Academy of Sciences, prehospital care has been in transition. Early research correlated survival with paramedic response, thus many systems quickly transitioned to a service with increasing number of ALS ambulances.

Methods: The Institutional Review Board (IRB) approved retrospective chart review of $14 \mathrm{EMS}$ provider agencies in the Dallas County area (population $>2,300,000$ ) for a year, from November 2012 - October 2013, looking at cardiac arrest and the success of Return of Spontaneous Circulation (ROSC) in this population.

Results: We analyzed 11,700 cardiac arrest calls during the time period, with 1,620 having a ROSC. Comparing BLS only vs single paramedic vs duel paramedic systems, $13.9 \%$ of dual systems had ROSC, $13.3 \%$ had ROSC that were single paramedic, with $0 \%$ having ROSC with a BLS only system.

Conclusion: ALS is important for ROSC during cardiac arrest; however, it is unclear if having two or more paramedic providers are necessary to achieve ROSC. If truly equivalent, then the cost savings of only having a single paramedic provider system might be worth looking into.

Prehosp Disaster Med 2017;32(Suppl. 1):s165

doi:10.1017/S1049023X17004459

\section{Prehospital Monitoring of Vital Parameters Using a Novel Device - RespiHeart \\ Johan Junker ${ }^{1}$, Carl-Oscar Jonson ${ }^{2}$ \\ 1. Center For Teaching And Research In Disaster Medicine And Traumatology, Linköping University, Linköping/Sweden \\ 2. Centre For Teaching And Research In Disaster Medicine And Traumatology, And Department Of Clinical And Experimental Medicine, Linköping University, Linköping/Sweden}

Study/Objective: The study aims at validating a novel device (RespiHeart) for monitoring vital parameters in traumatically injured patients.

Background: There is a need for a simple-to-use method for monitoring of vital parameters in the prehospital setting. RespiHeart is a small medical device that is attached to the sternum. It sends light of defined wavelengths into the underlying vasculature, and measures the reflected light. The resulting signals are then treated using proprietary algorithms to obtain heart rate and respiratory rate. The device has the capability to also measure oxygen saturation, temperature and movement.

Methods: The device was tested during training sessions for medical personnel, where various traumatic wounds were inflicted on anesthetized pigs. The training was primarily 